\title{
On the clustering of seismicity in the Southern Tyrrhenian area
}

\author{
Giuseppe Luongo and Adriano Mazzarella \\ Dipartimento di Geofisica e Vulcanologia, Università di Napoli «Federico II», Napoli, Italy
}

\begin{abstract}
High-quality shallow, intermediate and deep shocks from the Southern Tyrrhenian area (interval: 1985-1995) are analysed to draw inferences on the complex shape of the underlying Benioff zone. The hypothesis of an active NW-oriented subduction of the lithosphere, generated and stressed by the opening of the Tyrrhenian basin, is confirmed. The fractal behaviour of seismic data in its five-dimensional set (magnitude, time and space) is significantly identified, even if within specific ranges. The light but significant variations of the different fractal dimensions measured during more recent years is explained in terms of a reduction in the fracturing degree of the Southern Tyrrhenian crust.
\end{abstract}

Key words Benioff zone - fractals - scale invariance - clustering - integral correlation

\section{Introduction}

The spatial distribution of seismicity in the Southern Tyrrhenian area has been analysed many times with the aim of drawing inferences on the underlying complex Benioff zone located in the center of the Mediterranean Sea, a region characterized by complex tectonics and large-scale geodynamic deformations. The presence of deep earthquakes to a depth of 600 $\mathrm{km}$, the recent opening of the Tyrrhenian basin, the formation of the Appenninic chain, the shallow but strong seismic activity and the pervasive vulcanism indicate the complexity of a region undergoing a phase of fast and profound structural evolution (Anderson and Jack-

Mailing address: Dr. Adriano Mazzarella, Dipartimento di Geofisica e Vulcanologia, Università di Napoli «Federico II», Largo S. Marcellino 10, 80138 Napoli, Italy. son, 1987; Westaway, 1993; Hyppolyte et al., 1994; Milano et al., 1994; Giunchi et al., 1996; Neri et al., 1996). There are some discrepancies in the Benioff zone interpretation due to the poor quality of the data, the ambiguity of the interpretation of the scant spatial distribution of deep earthquakes and inadequate statistical methods (Caputo et al., 1970; McKenzie, 1972; Barberi et al., 1973; Gaudiosi et al., 1984; Iannaccone et al., 1985; Finetti and Del Ben, 1986; Locardi, 1986; Patacca and Scandone, 1989; Boccaletti et al., 1990; Mantovani et al., 1990; Luongo et al., 1991). The availability of an excellent new data set of shallow, intermediate and deep seismic events, taken from the Istituto Nazionale di Geofisica, Italy, and the computation of their fractal behaviour in its five-dimensional set (magnitude, time and space), inside specific ranges, allows a significant identification of the Benioff zone beneath the Southern Tyrrhenian Sea, dipping NW up to about $150 \mathrm{~km}$ and then dipping more and more $\mathrm{W}$ up to about $400 \mathrm{~km}$. In particular, the variations of the different fractal dimensions relative to the crustal earthquakes 
which occurred after 1990 would indicate a reduction in the fracturing degree of the Southern Tyrrhenian crust during more recent years.

\section{Data collection and analysis}

For the Southern Tyrrhenian area $\left(36-41^{\circ} \mathrm{N}\right.$; $12-18^{\circ} \mathrm{E}$; interval: $\left.1985-1995\right), 6773$ shallow, intermediate and deep seismic events, taken from the Istituto Nazionale di Geofisica, Italy, are analysed. The details of earthquake relocation on the basis of both the available number of $P$ - and $S$-readings are reported in Milano et al. (1994). For the estimation of the catalogue completeness, the magnitudes are grouped into intervals to account for the error due to the method of estimating the magnitude. After preliminary tests, an interval of 0.2 magnitude yielded the best-constrained shapes for the cumulative magnitude-frequency distribution. The data is found to be in full agreement with the Gutenberg-Ritcher relationship within the range $2.2 \leq m \leq 4.8$, with the slope $b=1.37 \pm 0.01$ (fig. 1). Eighty-eight percent of shocks are

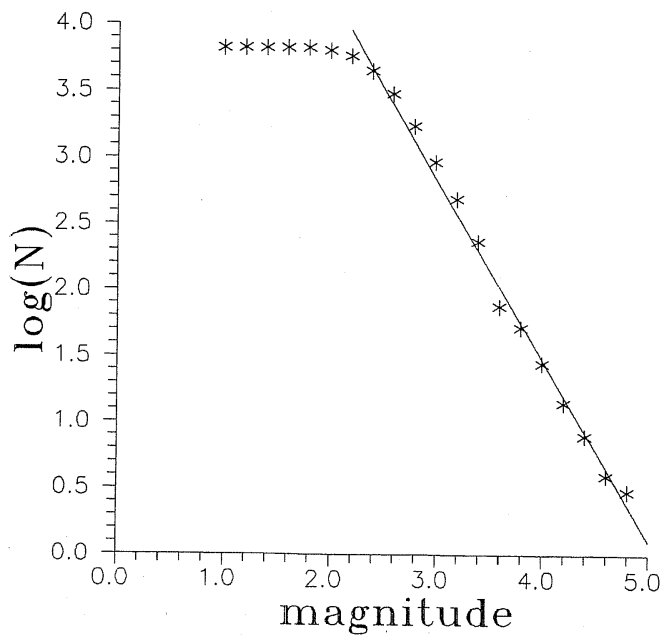

Fig. 1. Observed cumulative frequencies (star points) (with an increment of 0.2 magnitude) of earthquakes recorded in the Southern Tyrrhenian area in the 1985-1995 interval. The best fitting regression line of $\log (N)$ on magnitude is also reported. found to be distributed inside the first $25 \mathrm{~km}$. The identification of the underlying Benioff zone, along which the hypocenters are distributed, is computed according to a leastsquare regression plane of depth $Z(\mathrm{~km})$ on the corresponding latitude $X_{1}(\mathrm{~km})$ and longitude $X_{2}(\mathrm{~km})\left(X=a X_{1}+b X_{2}+c\right)$. The relative Dipping Angle DA (estimated in respect to a horizontal reference plane) and Dipping Orientation DO (estimated in respect to $\mathrm{W}$ reference orientation) of the investigated slab are obtained according to:

$$
\mathrm{DA}=\tan ^{-1} \sqrt{a^{2}+b^{2}}, \quad \mathrm{DO}=\tan ^{-1}(a / b) .
$$

The depth step is chosen to be equal to $25 \mathrm{~km}$ that is sufficiently large to account for error. The statistical significance level of such computation is obtained by testing the correlation coefficient $R$ against the hypothesis of zero correlation $\left(R^{2}\right.$ indicates the percentage of $Z$ variance accounted for by regression on $X_{1}$ and $X_{2}$ ): the regression plane identification is statistically significant at the $\alpha$ level when the following relationship is verified:

$$
\sqrt{(N-3)} / 2 \ln \left((1+R) /(1-R)>z_{\alpha / 2}\right),
$$

where $z_{\alpha / 2}$ is the standardized normal variable $(z=1.96$ for $\alpha=0.05$ and $z=2.58$ for $\alpha=0.01$ ) (Bendat and Piersol, 1971).

The timing of the seismic events (event times are typically known to within a minute) and the total length of the time available for the study (11 years) provide a wide range of available time-scales. This is why the time clustering of earthquakes is here investigated on the basis of the Cantor dust model (Mandelbrot, 1983; Luongo et al., 1996a,b). The interval $t_{0}$, over which the series of $N$ events occurs, is divided into a series of smaller $n$ intervals $t=t_{0} / n$ with $n=2,3,4 \ldots$ and the fraction $\mathrm{Pr}=N / n$ of intervals of length $t$, occupied by events, is computed. If the distribution of events has a fractal (power law) structure, then one obtains

$$
\operatorname{Pr} \sim t^{(1-D)}
$$


or, equivalently, on a log-log scaled plane

$$
\log (P r) \sim(1-D) \log (t) .
$$

$D$ is estimated from the regression coefficient of such a linear relationship, in a specific time range, when it is found to be confident at not less than $99 \%$ and to deviate significantly from the corresponding simulations obtained when the same number $N$ of events is equally or randomly spaced in time (Feller, 1968). The time range is the scale region over which the time structure of $N$ events is scale-invariant with a fractal dimension equal to $D$.

An earthquake can be considered a cluster of active sites and the hypocenter (epicenter) can be defined as the first site that becomes active in the three (two) dimensions. The spatial clustering of earthquakes is here investigated on the basis of the correlation integral $C(r)$ (Grassberger and Procaccia, 1983; Luongo et al., 1996a) so defined:

$$
C(r)=2 /(N(N-1)) \sum_{i=1}^{N} \sum_{\substack{J=1 \\ i \neq J}}^{N} \Theta\left(r-\left|X_{i}-X_{j}\right|\right),
$$

where $\Theta$ is the Heaviside function, $X_{i}$ is the coordinate set of the $i$-th earthquake. This correlation integral counts the number of pairs with distance $\left|X_{i}-X_{j}\right|$ smaller than $r$. This is done by taking each earthquake in turn as a center, and analysing the distribution of the remaining earthquakes relative to it. The normalization factor $2 /(N(N-1))$ represents the number of pairs so that $C(r)$ tends to 1 for $r$ tending to infinite. If the distribution of $N$ earthquakes has a fractal structure, then

$$
C(r) \sim r^{D}
$$

or, equivalently, on a log-log scaled plane

$$
\log (C) \sim D \log (r)
$$

$D$ is estimated from the regression coefficient of such a linear relationship, in a specific spatial range, when it is found to be confident at not less than $99 \%$ and to deviate significantly from the correspondent simulations when the same number $N$ of events is equally or randomly spaced (Feller, 1968). The spatial range represents the scale-length of distance over which the clustering of earthquakes is scale invariant with a fractal dimension equal to $D$.

\section{Results}

The coefficients of the different regression planes, fitting the hypocenters at an ever deeper level (with a step of $25 \mathrm{~km}$ ) and found to be confident at a level not less than $95 \%$, are reported in table I, together with the relative correlation coefficient $R$; the dipping angles and orientations of such planes for increasing depth are reported in fig. 2a,b. A very complex Benioff zone appears, with a NW dipping angle increasing up to $50^{\circ}$ at a depth of $150 \mathrm{~km}$ and with a more and more $\mathrm{W}$ dipping angle gradually decreasing for increasing depths.

To identify the specific time-range in which the earthquakes follow the power law (2.1) and to quantify the degree of time clustering of the earthquakes, the fraction $R$ of time intervals including an event was plotted on log-log scale as a function of the interval size $t$. The smallest time interval was chosen to be $1 \mathrm{~min}$. The fractal dimension $D=1-s$ was estimated from the slope $s$ that provides the best fitting of least squares regression line of $\log (P r)$ on $\log (t)$ over a particular time range. The results show that for all the 5002 available earthquakes occurring inside the first $25 \mathrm{~km}$ and enclosed in the range of magnitude $(2.2 \leq m \leq 4.8)$ for which the catalogue is complete, the best fitting of the least-squares regression line of $\log (P r)$ on $\log (t)$ (significantly different from the relative random and uniform simulations and confident at a level higher than 99\%) was obtained for time intervals ranging from $16 \mathrm{~min}$ to about $34 \mathrm{~h}$, with a fractal dimension equal to $0.18 \pm 0.01$ (fig. 3). For $t>34 \mathrm{~h}$, the curvature of the log-log plot did not allow a direct interpretation of the fractal dimension; for $t>11$ days, every interval contained an earthquake $(D=1)$. For the 707 earthquakes deeper than $25 \mathrm{~km}$, the time clustering regime was not significantly different from the relative random and uniform simulations. 
Table I. Parameters of the least square regression plane of depth $Z(\mathrm{~km})$ on the corresponding latitude $X_{1}(\mathrm{~km})$ and longitude $X_{2}(\mathrm{~km})\left(Z=a X_{1}+b X_{2}+c\right)$. $N$ is the number of events and $R$ is the relative correlation coefficient whose square indicates the percentage of $Z$ variance accounted for by regression on $X_{1}$ and on $X_{2}$.

\begin{tabular}{lcccc}
\hline \hline & $a$ & $b$ & $N$ & $R$ \\
\hline$Z \geq 25 \mathrm{~km}$ & 0.46 & -0.47 & 707 & 0.44 \\
$Z \geq 50 \mathrm{~km}$ & 0.57 & -0.52 & 566 & 0.50 \\
$Z \geq 75 \mathrm{~km}$ & 0.75 & -0.59 & 480 & 0.60 \\
$Z \geq 100 \mathrm{~km}$ & 0.86 & -0.73 & 410 & 0.48 \\
$Z \geq 125 \mathrm{~km}$ & 0.76 & -0.73 & 319 & 0.67 \\
$Z \geq 150 \mathrm{~km}$ & 0.63 & -0.83 & 237 & 0.67 \\
$Z \geq 175 \mathrm{~km}$ & 0.44 & -0.70 & 169 & 0.58 \\
$Z \geq 200 \mathrm{~km}$ & 0.30 & -0.63 & 137 & 0.48 \\
$Z \geq 225 \mathrm{~km}$ & 0.41 & -0.83 & 118 & 0.58 \\
$Z \geq 250 \mathrm{~km}$ & 0.36 & -0.77 & 95 & 0.56 \\
$Z \geq 275 \mathrm{~km}$ & 0.28 & -0.79 & 69 & 0.58 \\
$Z \geq 300 \mathrm{~km}$ & 0.04 & -0.79 & 37 & 0.55 \\
$Z \geq 325 \mathrm{~km}$ & 0.07 & -0.70 & 25 & 0.49 \\
$Z \geq 350 \mathrm{~km}$ & -0.07 & -0.68 & 15 & 0.58 \\
$Z \geq 375 \mathrm{~km}$ & -0.16 & -0.60 & 10 & 0.64 \\
\hline
\end{tabular}

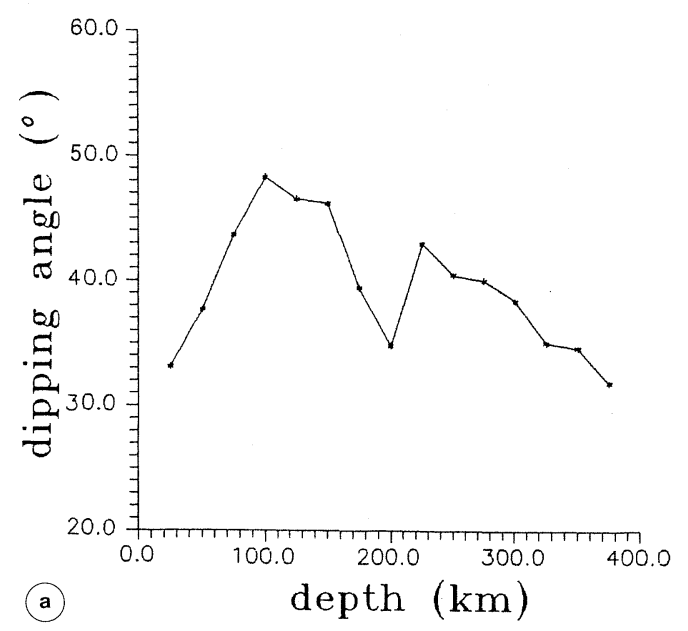

Fig. 2a,b. a) Dipping angle (estimated with respect to a horizontal reference plane); b) dipping orientation (estimated with respect to a $\mathrm{W}$ reference orientation) of the Tyrrhenian Benioff zone as a function of depth $(\mathrm{km})$.

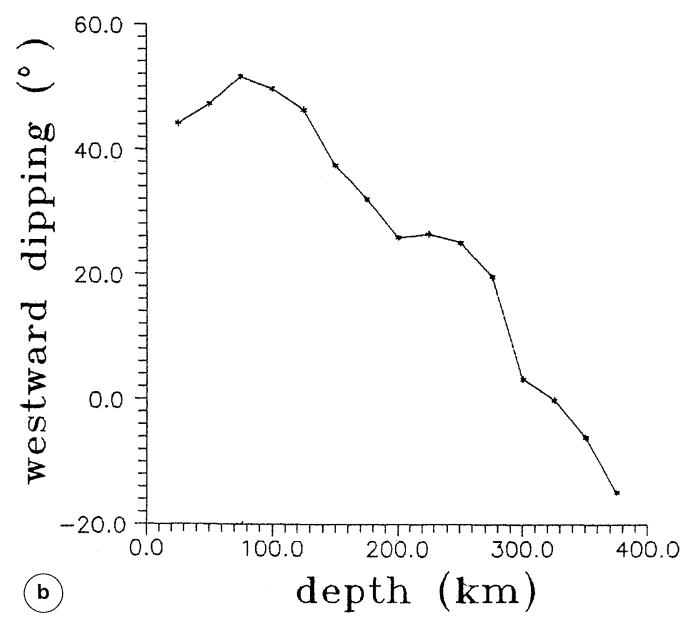




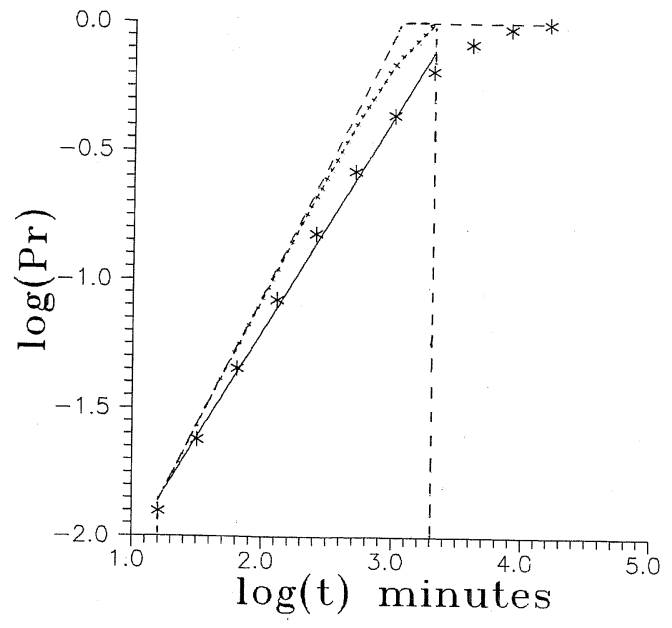

Fig. 3. The $\log$ of the fraction $\operatorname{Pr}$ of time intervals of length $t$ (in minutes) including the seismic events, within the range of magnitude $2.2 \leq m \leq 4.8$ as a function of $\log (t)$. Star points are related to the observed data while the crossed and dashed lines represent the relative random and uniform simulations, respectively. Vertical dashed lines represent the lower and upper limits of $\log (P r)$ inside which the linear slope provides the best fitting to the observed data.

To quantify the degree of spatial clustering of earthquakes and identify the specific spatial range in which the earthquakes follow the power law (2.2), the number of earthquakes $C$ with distance smaller than $r$ was plotted on $\log$-log scale as a function of distance $r$. The smallest distance was chosen to be $1 \mathrm{~km}$. The fractal dimension of epicenter $D_{e}$ and of hypocenter distribution $D_{h}$ was estimated directly from the slope that provides the best fitting of least squares regression line of $\log (C)$ on $\log (r)$. The fractal spatial analysis of the 5002 shallow earthquakes occurring inside the first $25 \mathrm{~km}$ provides results similar to those obtained for an epicentral distribution owing to the smallness of the depth with respect to the other two dimensions. The best fitting of the least squares regression line of $\log (C)$ on $\log (r)$ (significantly different from the relative random and uniform simulations and confident at a level higher than 99\%) was obtained for

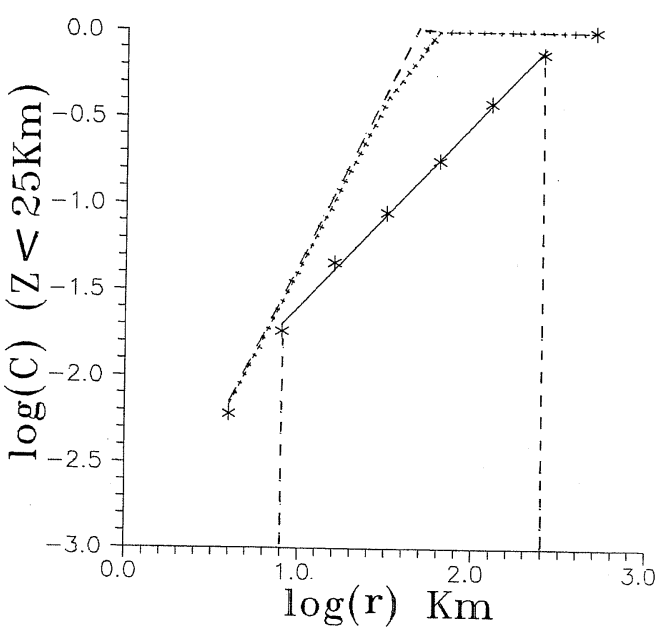

Fig. 4. The $\log$ of the number of pairs $C$ of epicenter distribution with distance smaller than $r$, within the range of magnitude $2.2 \leq m \leq 4.8$, as a function of the $\log$ of the distance $r(\mathrm{~km})$. Star points are related to the observed data while the crossed and dashed lines represent the relative random and uniform simulations, respectively. Vertical dashed lines represent the lower and upper limits of $\log (C)$ inside which the linear slope provides the best fitting to the observed data

distances from $8 \mathrm{~km}$ to $256 \mathrm{~km}$ with the fractal dimension $D_{e}=1.05 \pm 0.01$. The curvature of $\log -\log$ plot for $r>256 \mathrm{~km}$ did not allow a direct interpretation of the fractal dimension $D_{e}$; all the epicenters were found to occur inside a distance of $510 \mathrm{~km}$ that is the diameter of the maximum circle inside the investigated Tyrrhenian region (fig. 4). As $r$ approaches such a diameter there are fewer and fewer pairs whose separation is as large as the diameter. Thus, for values of $r$ greater than the size of the investigated area, no pair is found, and the number of pairs with distances less than $r$ remains the same as $r$ increases (the edge effect). For the 707 earthquakes deeper than $25 \mathrm{~km}$, the best fitting of the least square regression line of $\log (C)$ on $\log (r)$ (significantly different from the relative random and uniform distributions and confident at a level higher than $99 \%$ ) is obtained for distances up to $70 \mathrm{~km}$ with a fractal dimension $D_{e}=1.82 \pm 0.01$ and 


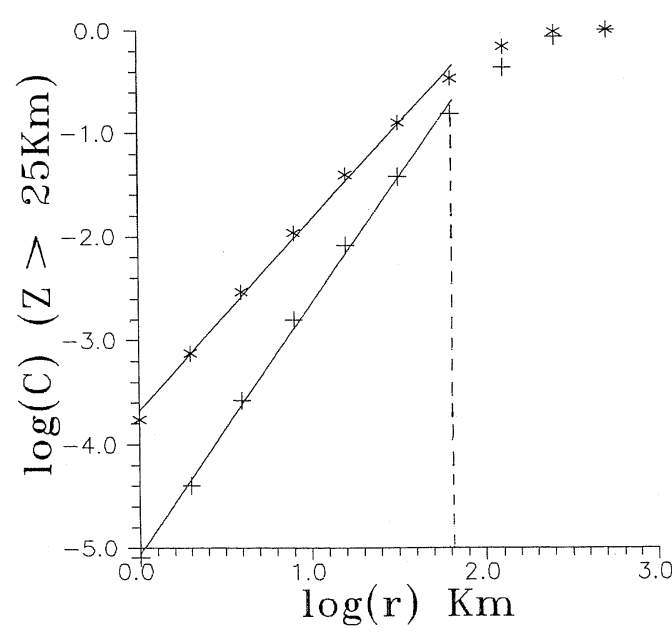

Fig. 5. The log of the number of pairs $C$ of epicenter (star points) and hypocenter (cross points) distribution deeper than $25 \mathrm{~km}$ with distance smaller than $r$, within the range of magnitude $2.2 \leq m \leq 4.8$, as a function of the $\log$ of the distance $r(\mathrm{~km})$. The vertical dashed line represents the upper limit of $\log (C)$ inside which the linear slope provides the best fitting to the observed epicenter and hypocenters.

$D_{h}=2.40 \pm 0.01$ (fig. 5); for the 410 earthquakes deeper than $100 \mathrm{~km}$, the best fitting of the least square regression line of $\log (C)$ on $\log (r)$ is obtained again for distances up to $70 \mathrm{~km}$ but with a fractal dimension $D_{e}=1.80 \pm 0.01$ and $D_{h}=2.44 \pm 0.01$.

\section{Discussion}

The Benioff zone beneath the Southern Tyrrhenian Sea is oriented almost entirely in the NW direction up to $150 \mathrm{~km}$ and then more and more oriented in the $\mathrm{W}$ direction (fig. 2a,b). The dipping-angle breaks in correspondence with $100 \mathrm{~km}$ and $200 \mathrm{~km}$ are coherent with a geodynamic model in which the stress field acting in Southern Italy is governed by the opening of the Tyrrhenian Basin (Luongo et al. 1991; Milano et al., 1994). Each fractal dimension obtained in the analysis of the five-dimensional set of earthquakes (magnitude, time, space) represents a particular aspect of the fractal structure of the fracturing process occurring in the Southern Tyrrhenian Sea and therefore need not be equal. The magnitude distribution follows the scaling law only within the range $2.2 \leq m \leq 4.8$. The upper limit corresponds to a depth of about $350 \mathrm{~km}$ that is an important structural interface separating materials with different elastic response where asymmetrical fault systems do not follow the scaling rules of fractal geometry. The fractal dimension with respect to magnitude is simply twice the $b$ value $(D=2 b)$ as a necessary consequence of the fractal distribution of fault areas (Luongo et al. 1996a,b). The obtained value of $D=2.74$ shows a low degree of clustering along fault planes. The time distribution of earthquakes shows a large fractal degree of clustering $(D=0.18)$ only within the interval ranging from $16 \min$ to $34 \mathrm{~h}$; for $t>34 \mathrm{~h}$ a kind of saturation begins to occur up to 11 days where each earthquake occurs with a probability of 1 (fig. 3).

The epicenters occurring inside the first $25 \mathrm{~km}$ are distributed along a line $\left(D_{e}=1.05\right)$ for distances ranging from $8 \mathrm{~km}$ to $256 \mathrm{~km}$. The earthquakes deeper than $25 \mathrm{~km}$ are also distributed with self-similar structure along very irregular surfaces and lines, respectively, up to distances in the order of $70 \mathrm{~km}\left(D_{e}=1.8\right.$, $D_{h}=2.4$ ); for distances $>70 \mathrm{~km}$ a kind of saturation begins to occur owing to the edge geometric effects related to the finiteness of the seismic volume investigated. The dimension of a planar section of a fractal set with fractal dimension $D$ in $3 D$ space is $D-1$ when the depth of earthquakes is not related to surface coordinates, i.e., when the spatial distribution of point sources is completely symmetric (Mandelbrot, 1983). So, a distribution of hypocenters along a horizontal plane is related to a value of $D_{h}-D_{e}=0$, while a distribution of hypocenters along a vertical plane is related to a value of $D_{h}-D_{e}=1$. The ascertained difference of 0.6 between the two fractal dimensions indicates a distribution of earthquakes along an oblique slab typical of a Benioff zone. The fractal analysis in its five-dimensional set was also applied to two sub-catalogues of crustal earthquakes $(Z \leq 25 \mathrm{~km})$ relative to non-overlapping intervals: 1985-1990, 1991- 
Table II. Values of fractal dimension with respect to magnitude, time of occurrence and areal distribution for two sub-catalogues of shallow earthquakes (less than $25 \mathrm{~km}$ ) relative to two non-overlapping intervals.

\begin{tabular}{lcc}
\hline \hline \multicolumn{1}{c}{ Interval } & $1985-1990$ & $1991-1996$ \\
\hline Magnitude & 1.5 & 1.4 \\
Time of occurrence & 0.1 & 0.2 \\
Areal distribution & 0.9 & 1.0 \\
\hline
\end{tabular}

1996. It appears that the fractal dimension relative to the more recent period decreases with respect to magnitude but increases with respect both to time of occurrence and to areal distribution (table II). All these features are coherent with a scenario of reduced fracturing of the Tyrrhenian crust according to the results obtained in the laboratory when system loading is decreasing (Hirata, 1987; Hirata et al., 1987). A detailed knowledge of the clustering fractal structure of the seismic distribution with respect to magnitude, time and space, might allow a better understanding of seismic processes and possibly assist in seismic predictions.

\section{REFERENCES}

ANDERSON, H. and J. JACKSON (1987): The deep seismicity of the Tyrrhenian Sea, Geophys. J. R. Astron. Soc., 91, 613-637.

BARberi, F., P. GASPARINI, F. INNOCENTI and L. VILlari (1973): Volcanism of the Tyrrhenian Sea and its geodynamics implications, J. Geophys. Res., 78, 5221-5232.

Bendat, J.S. and A.C. Piersol (1971): Random Data: Analysis and Measurement Procedures (Wiley Interscience, New York), 125-133.

BocCAletti, M., R. NiCOLICH and L. TORTORICI (1990): New data and hypothesis on the development of the Tyrrhenian basin, Palaeogeogr., Palaeoclimatol., Palaeocol., 77, 15-40.

CAPuto, M., G.F. Panza and D. PostPischl (1970): Deep structure of the Mediterranean basin, J. Geophys. Res., 75, 4919-4923.

FELLER, W. (1968): An Introduction to Probability Theory and its Applications (John Wiley, New York), vol. 1, $101-106$

FinetTI, I. and A. DeL BeN (1986): Geophysical study of the Tyrrhenian opening, Boll. Geofis. Teor. Appl., 27, 75-155.

Gaudiosi, G., G. LuONGo and G.P. Ricciardi (1984): A bending model for the Calabrian arc, in Problems and Methods for Litospheric Exploration, edited by R. CASsinIs (Plenum Publishing Corporation), 191-199.

Giunchi, C., R. SABAdini, E. Boschi and P. Gasperini (1996): Dynamic models of subduction. Geophysical and geological evidence in the Tyrrhenian Sea, Geophys. J. Int., 126, 555-578.

Grassberger, P. and I. ProcaCcia (1983): Characterization of strange attractors, Phys. Rev. Lett., 50, 346-349.

Hirata, T. (1987): Omori's power law aftershock sequences of microfracturing in rock fracture experiment, J. Geophys. Res., 92, 6215-6221.

Hirata, T., T. SATOH and K. ITO (1987): Fractal structure of spatial distribution of microfracturing in rock, Geophys. J. R. Astron. Soc., 90, 369-374.

Hyppolyte, J.C., J. ANGERlier and F. Roure (1994): A major geodynamic change revealed by Quaternary stress patterns in the Southern Appennines (Italy), Tectonophysics, 230, 199-210.

IANNACCONE, G., G. SCARCElla and R. ScARPa (1985): Subduction zone geometry and stress pattern in the Tyrrhenian Sea, Pageoph, 123, 819-836.

LOCARDI, E. (1986): Tyrrhenian volcanic arcs: volcanotectonics, petrogenesis and economic aspects, in The Origin of Arcs, edited by F.C. WEZEL (Elsevier, Amsterdam), 351-373.

Luongo, G., E. Cubellis, F. Obrizzo and S.M. PetrazZUOLI (1991): A physical model for the origin of volcanism of the Tyrrhenian margin: the case of Neapolitan area, J. Volcanol. Geotherm. Res., 48, 173-185.

Luongo, G., A. Mazzarella and A. Palumbo (1996a): On the self-organized critical state of Vesuvio volcano, J. Volcanol. Geotherm. Res., 70, 76-73.

Luongo, G., A. Mazzarella and A. Palumbo (1996b): A fractal approach to clustering of the 1983-1984 seismicity in the Campi Flegrei caldera, Southern Italy, Fractals, 4, 29-34.

MANDELBROT, B.B. (1983): The Fractal Geometry of Nature (Freeman, New York), p. 468.

Mantovani, E., D. BabbuCCI, D. Albarello and M. MuCCIARELli (1990): Deformation pattern in the Central Mediterranean and behavior of the African/Adriatic promontory, Tectonophysics, 179, 63-79.

McKenzIE, D. (1972): Active tectonics of the Mediterranean region, Geophys. J. R. Astron. Soc., 30, 109-185.

Milano, G., G. Vilardo and G. LuONGo (1994): Continental collision and basin opening in Southern Italy: a new plate subduction in the Tyrrhenian Sea?, Tectonophysics, 230, 249-264.

Neri, G., D. Caccamo, O. Cocina and A. Montalto (1996): Geodynamic implications of earthquake data in the Southern Tyrrhenian Sea, Tectonophysics, 258, 233-249.

Patacca, E. and P. SCANDOne (1989): Post-Tortonian mountain building in the Appennines. The role of the passive sinking of a relic lithospheric slab, in The Lithosphere in Italy. Advances in Earth Science Research, edited by A. BORIANI, M. BONAFEDE, G.B. PICCARDo and G.B. VAI, Atti Acc. Naz. Lincei, Roma, 80, 157-176.

WESTAWAY, R. (1993): Quaternary uplift of Southern Italy, J. Geophys. Res., 98, 21741-21772. 